\title{
Forecasting COVID-19 with Importance Sampling and Path-Integrals
}

By Lester Ingber

Physical Studies Institute LLC

Abstract-Background: Forecasting nonlinear stochastic systems most often is quite difficult, without giving in to temptations to simply simplify models for the sake of permitting simple computations.

Objective: Here, two basic algorithms, Adaptive Simulated Annealing (ASA) and path-integral codes PATHINT/PATHTREE (and their quantum generalizations qPATHINT/ qPATHTREE) are suggested as being useful to fit COVID-19 data and to help predict spread or control of this pandemic. Multiple variables are considered, e.g., potentially including ethnicity, population density, obesity, deprivation, pollution, race, environmental temperature.

Method: ASA and PATHINT/PATHTREE have been demonstrated as being effective to forecast properties in three disparate disciplines in neuroscience, financial markets, and combat analysis.

Results: Not only can selected systems in these three disciplines be aptly modeled, but results of detailed calculations have led to new results and insights not previously obtained.

Keywords: path integral, importance sampling, financial options, combat analysis.

GJHSS-H Classification: FOR Code: 111799

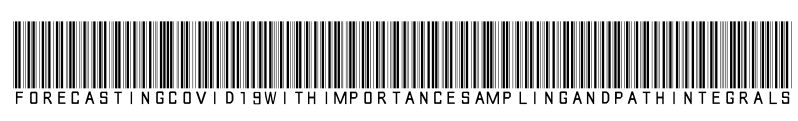

Strictly as per the compliance and regulations of:

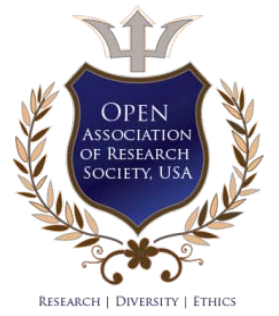

(C) 2021. Lester Ingber. This research/review article is distributed under the terms of the Attribution-NonCommercial-NoDerivatives 4.0 International (CC BY-NC-ND 4.0). You must give appropriate credit to authors and reference this article if parts of the article are reproduced in any manner. Applicable licensing terms are at https://creativecommons.org/licenses/by-nc-nd/ 4.0/. 


\title{
Forecasting COVID-19 with Importance Sampling and Path-Integrals
}

\author{
Lester Ingber
}

Abstract- Background: Forecasting nonlinear stochastic systems most often is quite difficult, without giving in to temptations to simply simplify models for the sake of permitting simple computations.

Objective: Here, two basic algorithms, Adaptive Simulated Annealing (ASA) and path-integral codes PATHINT/PATHTREE (and their quantum generalizations qPATHINT/qPATHTREE) are suggested as being useful to fit COVID-19 data and to help predict spread or control of this pandemic. Multiple variables are considered, e.g., potentially including ethnicity, population density, obesity, deprivation, pollution, race, environmental temperature.

Method: ASA and PATHINT/PATHTREE have been demonstrated as being effective to forecast properties in three disparate disciplines in neuroscience, financial markets, and combat analysis.

Results: Not only can selected systems in these three disciplines be aptly modeled, but results of detailed calculations have led to new results and insights not previously obtained.

Conclusion: While optimization and path-integral algorithms are now quite well-known (at least to many scientists), these applications give strong support to a quite generic application of these tools to stochastic nonlinear systems.

Keywords: path integral, importance sampling, financial options, combat analysis.

\section{InTRODUCTION}

$t$ is generally recognized that the spread of COVID-19 is affected by multiple variables, e.g., potentially including ethnicity, population density, obesity, deprivation, pollution, race, environmental temperature (Anastassopoulou et al, 2020; Bray et al, 2020; Li et al, 2020). Also, the Centre for Evidence-Based Medicine (CEBM) regularly cites papers on the dynamics of COVID-19 at https://www.cebm.net/evidence-synthesis/ transmission-dynamics-of-covid-19/.

This proposal offers the application of two basic multivariate algorithms to fairly generic issues in forecasting. As such, they may be useful to fit COVID19 data and to help predict upcoming spread and control of this pandemic.

(a) Adaptive Simulated Annealing (ASA) developed by the author (Ingber, 1993a) is an importancesampling optimization code usually used for nonlinear, nonequilibrium, non-stationary, multivariate systems.

Author: Physical Studies Institute LLC, Ashland, OR, USA. e-mail: ingber@caa.caltech.edu (b) PATHINT is a numerical path-integral PATHINT code developed by the author (Ingber, 1993b) used for propagation of nonlinear probability distributions, including discontinuities.

These codes were developed by the author and applied across multiple disciplines.

There is not "one size fits all" in forecasting different systems. This was demonstrated for three systems (Ingber, 2020b), where the author has addressed multiple projects across multiple disciplines using these tools: 72 papers/reports/lectures in neuroscience, e.g. (Ingber, 2018; Ingber, 2021), 31 papers/reports/lectures in finance, e.g. (Ingber \& Mondescu, 2003; Ingber, 2020a), 24 papers/reports/ lectures in combat analyses, e.g. (Ingber, 1993b; Ingber, 2015), and 11 papers/reports/lectures in optimization, e.g. (Atiya et al, 2003; Ingber, 2012), It is reasonable to expect that this approach can be applied to many other projects.

For example, the path-integral representation of multivariate nonlinear stochastic differential equations permits derivation of canonical momenta indicators (CMI) which are faithful to intuitive concepts like Force, Momenta, Mass, etc (Ingber, 1996; Ingber, 2015; Ingber \& Mondescu, 2001). Correlations among variables are explicitly included in the CMI.

\section{DATA}

A large and updated database for COVID-19 is maintained by the John Hopkins University (JHU) at https://github.com/CSSEGISandData/COVID-19/blob/m aster/archived_data/archived_daily_case_up-dates/0121-2020_2200.csv. This database was used for a pilot study.

\section{a) 50+ Locations}

The data being used contains 3340 cities throughout the US and some territories. The locations have been broken into 57 States and Territories ready for production runs.

\section{ili. Technical Considerations}

If there is not time to process large data sets, then the data can be randomly sampled, e.g., as described in another paper, "Developing bid-ask probabilities for high-frequency trading" (Ingber, 2020a). 
If the required forecast is longer than the conditional distribution can sustain, PATHINT/ PATHTREE can be used to propagate the distribution.

The dataset should be broken into independent Training and Testing subsets, to test the trained distribution. If this is not possible, e.g., because of data or time limitations, at the least experts can be used to judge if the model is ready for realtime applications, e.g., the Delphi method (Okoli \& Pawlowski, 2004).

If an algorithm like ASA is to be used across a large class of problems, then it must be tunable to different classes. Over the $30+$ years of ASA development, the author has worked with many volunteers who have contributed valuable ideas, modifications and corrections to this code. This has resulted in over 150 ASA options that can be used for additional timing additional tuning making it useful across many classes of problems.

The path integral algorithm includes its mathematical equivalents, a large class of stochastic differential equations and a large class of partial differential equations. The advantages of the path integral algorithm are:

(a) Intuitive description in terms of classical forces, inertia, momentum, etc., leading to new indicators.

(b) Delivering a cost function derived from a Lagrangian, or its Action (Lagrangian $x d t$ ). Sometimes constraints need to be added as Lagrange multipliers, as was required for normalization requirements in financial risk projects (Ingber, 2010).

\section{Pilot Study}

The shape of the spread of this virus is clearly nonlinear. A simple model was used for a pilot study to at least capture some nonlinearity. For example, just using the daily number of total cases reported, $C$, the short-time conditional Probability $P(t+1 \mid t)$ is given in terms of its effective Lagrangian $L, P=$ $\exp \left(L_{\text {eff }} d t\right)$ (including the logarithm of the prefactor normalization as it may contain nonlinearities as modeled here):

$$
\begin{aligned}
& L_{\text {eff }}=\left[\left(x_{t+1}-x_{t}-g_{x} d t\right) g_{x x^{\prime}}\left(x_{t+1}^{\prime}-x_{t}^{\prime}-g_{x^{\prime}} d t\right)+1 / 2 \log \left(2 \pi d t g^{2}\right)\right. \\
& g_{x}=a \exp \left(x^{b}\right) \\
& g_{x x^{\prime}}=c \exp \left(x^{d}\right) \\
& g=\operatorname{det}\left(g_{x x^{\prime}}\right)
\end{aligned}
$$

with parameters to be fit to data $\{a, b, c, d\}$. This is a simple one-factor model. In more than one dimension, $g_{x x}$ is the metric of this space, the inverse of the covariance matrix.

For the full data set, 100,000 generated-state iteration-s of this cost/objective function's states over the $\mathrm{JHU}$ data gave

$$
a=0.077, b=0.874, c=2.79, d=0.845
$$

\section{a) Comet Profile}

These codes were run on XSEDE Comet, for 100000 generated states.

"Comet is a dedicated XSEDE cluster designed by Dell and SDSC delivering 2.0 petaflops, featuring Intel next-gen processors with AVX2, Mellanox FDR InfiniBand interconnects and Aeon storage. The standard compute nodes consist of Intel Xeon E5-2680v3 (formerly codenamed Haswell) processors, 128

GB DDR4 DRAM (64 GB per socket), and 320 GB of SSD local scratch memory. The GPU nodes contain four NVIDIA GPUs each. The large memory nodes contain 1.5 TB of DRAM and four Haswell processors each. The network topology is 56 Gbps FDR InfiniBand with rack-level full bisection bandwidth and 4:1 oversubscription cross-rack bandwidth. Comet has 7 petabytes of $200 \mathrm{~GB} /$ second performance storage and 6 petabytes of $100 \mathrm{~GB} / \mathrm{second}$ durable storage. It also has dedicated gateway hosting nodes and a Virtual Machine repository. External connectivity to Internet2 and ESNet is 100 Gbps."

Comet is being phased out and users will soon be using the new Expanse platform.

\section{b) Parallel Processing}

"Parallel Processing for this project basically is similar to many projects developed by the author as Principal Investigator at the Extreme Science and Engineering Discovery Environment (XSEDE.org) since February 2013. That is "trivial MPI" is used, wherein many simultaneous runs are achieved by simply reading in different data files to ASA, using the "array" feature offered by some XSEDE platforms. As offered in a previous XSEDE Extended Collaborative Support Service (ECSS) ticket:

Parallelization efficiency is 1 for jobs running on a single core that is max one could get. For multi-threaded apps one can get some to decent bump in speed using multiple cores up to some point before plateauing. However, speed bump with multiple cores often leads drop in parallelization efficiency.

Drawback of using single core is too long run time. Though in this case, you are running array jobs with single core and getting maximum efficiency. This is the ideal situation on 'Comet' because nodes on this machine can be shared. You should explain on Scaling and parallelization efficiency 
section that your application is not multi-threaded and you use single core on comet to run your jobs. This gives efficiency of 1 , which is maximum value achievable. However, you run array of jobs in one submission and each job uses a single core. This is most efficient use of resources because node sharing is allowed on Comet. It won't hurt to write that you have consulted XSEDE staff on this matter."

\section{c) Xeon Processor}

The full US run was done on the author's P1 Gen 3 Thinkpad with a Xeon processor. Previous runs show full agreement between the Comet and the Thinkpad runs when "-ffloat-store" is added to the compile parameters. A full US run of 100,000 generated states with 3239 non-zero locations took $1 \mathrm{hr} 47 \mathrm{~min} 17$ sec. (All runs including subsets of the full US therefore took about twice that long.)

\section{All Results}

All locations were processed to exclude those with all "0" for all days, 99 of them.

Note that a few locations, those with just sublocation as it turned out, gave parameter values that hit boundaries of assigned parameter maximums or minimums. Since these were few exceptions, the decision was made to keep the default ranges given in Table 1.

\section{Table 1}

$\begin{array}{ccc}\text { Par } & \text { Min } & \text { Max } \\ 0 & -2 & 2 \\ 1 & -2 & 2 \\ 2 & 0.1 & 2 \\ 3 & -2 & 2\end{array}$

Final Results for all 58 Locations are given in Table 2.

\section{Table 2}

RUNS COVID/asa usr out 01-Alabama final cost value $=\overline{0} .00 \overline{0} 616 \overline{5} 903$

Parameter Value

$$
\begin{array}{cc}
0 & 0.07526909 \\
1 & 0.7867917 \\
2 & 0.1 \\
3 & 1.036661
\end{array}
$$

RUNS_COVID/asa usr out 02-Alaska final cost value $=\overline{0.0008660421}$

Parameter Value

$$
\begin{array}{cc}
0 & 0.03041555 \\
1 & 0.9221085 \\
2 & 0.1 \\
3 & 0.9276368
\end{array}
$$

RUNS_COVID/asa_usr_out_03-Arizona

final cost value $=\overline{0} .00 \overline{3} 91 \overline{2} 767$

Parameter Value

$$
\begin{array}{cc}
0 & 0.08377208 \\
1 & 0.818453 \\
2 & 0.1 \\
3 & 1.287453
\end{array}
$$

RUNS_COVID/asa_usr_out 04-Arkansas final cost value $=0.0004816542$

Parameter Value

$$
\begin{array}{cc}
0 & 0.07941101 \\
1 & 0.7750893 \\
2 & 0.1 \\
3 & 1.183597
\end{array}
$$

RUNS COVID/asa usr out 05-California final cost value $=0.0009490655$

Parameter Value

$$
\begin{array}{cc}
0 & 0.06696538 \\
1 & 0.8527078 \\
2 & 0.1 \\
3 & 1.292374
\end{array}
$$

RUNS_COVID/asa_usr_out_06-Colorado final cost value $=\overline{0} .00050 \overline{3} 892$

Parameter Value

$$
\begin{array}{cc}
0 & 0.02576714 \\
1 & 0.875757 \\
2 & 0.1076587 \\
3 & 1.033743
\end{array}
$$

RUNS_COVID/asa_usr_out_07-Connecticut final cost value $=\overline{0} .006819112$

Parameter Value

$$
\begin{array}{cc}
0 & 0.03883795 \\
1 & 0.7877583 \\
2 & 0.1499112 \\
3 & 1.133882
\end{array}
$$

RUNS_COVID/asa_usr_out_08-Delaware final cost value $=\overline{0} .00 \overline{4} 94 \overline{9} 477$

Parameter Value

$$
\begin{array}{cc}
0 & 0.1227538 \\
1 & 0.6899159 \\
2 & 0.261152 \\
3 & 0.9695861
\end{array}
$$

RUNS_COVID/asa_usr_out_09-Diamond_Princess final cost value $=-0.05391078$

$\begin{array}{cc}\text { Parameter } & \text { Value } \\ 0 & -4.98784 \mathrm{e}-07 \\ 1 & -1.992295 \\ 2 & 0.1 \\ 3 & -2\end{array}$


RUNS_COVID/asa_usr_out_10-District_of_Columbia final cost value $=\overline{0} 0.06 \overline{6} 929 \overline{9} 41$

Parameter value

$$
\begin{array}{cc}
0 & 2 \\
1 & 0.4060976 \\
2 & 2 \\
3 & 0.7794162
\end{array}
$$

RUNS COVID/asa usr out 11-Florida final cost value $=\overline{0} .000 \overline{8} 10 \overline{1} 027$

Parameter Value

$$
\begin{array}{cc}
0 & 0.0844608 \\
1 & 0.8210241 \\
2 & 0.1 \\
3 & 1.270596
\end{array}
$$

RUNS_COVID/asa_usr_out_12-Georgia final cost value $=\overline{0} .000264 \overline{3} 592$

Parameter Value

$$
\begin{array}{cc}
0 & 0.04424673 \\
1 & 0.8548552 \\
2 & 0.1 \\
3 & 1.162738
\end{array}
$$

RUNS_COVID/asa_usr_out_13-Grand_Princess final cost value $=-{ }^{-}-\overline{0} 063622$

$$
\begin{array}{ll}
\text { Parameter } & \text { Value } \\
0 & -6.538724 \mathrm{e}-08 \\
1 & -1.806268 \\
2 & 0.1 \\
3 & -2
\end{array}
$$

RUNS_COVID/asa_usr_out_14-Guam final cost value $=\overline{0} 0.0465182$

Parameter Value

$$
\begin{array}{cc}
0 & 0.008877227 \\
1 & 1.154289 \\
2 & 0.1 \\
3 & 1.147461
\end{array}
$$

RUNS_COVID/asa_usr_out_15-Hawaii final cost value $=\overline{0} .00 \overline{6} 86 \overline{2} 005$

Parameter Value

$$
\begin{array}{cc}
0 & 0.01611866 \\
1 & 1.050401 \\
2 & 0.1 \\
3 & 1.102763
\end{array}
$$

RUNS_COVID/asa_usr_out_16-Idaho final cost value $=\overline{0} .000748 \overline{8} 098$

Parameter Value

$$
\begin{array}{cc}
0 & 0.05115676 \\
1 & 0.8504985 \\
2 & 0.1 \\
3 & 1.084733
\end{array}
$$

RUNS_COVID/asa_usr_out_17-Illinois final cost value $=\overline{0} .000 \overline{4} 48 \overline{1} 785$

Parameter Value

$$
\begin{array}{cc}
0 & 0.06157631 \\
1 & 0.8171975 \\
2 & 0.8193241 \\
3 & 1.021197
\end{array}
$$

RUNS_COVID/asa usr_out_18-Indiana final cost value $=\overline{0} .000 \overline{3} 78 \overline{7} 652$

Parameter Value

$$
\begin{array}{cc}
0 & 0.0412226 \\
1 & 0.8332504 \\
2 & 0.1 \\
3 & 0.9823153
\end{array}
$$

RUNS COVID/asa usr out 19-lowa final cost value $=\overline{0} .000 \overline{3} 52 \overline{5} 547$

Parameter Value

$$
\begin{array}{cc}
0 & 0.07068677 \\
1 & 0.7683947 \\
2 & 0.1387974 \\
3 & 1.049687
\end{array}
$$

RUNS_COVID/asa usr out 20-Kansas final cost value $=\overline{0} .000 \overline{2} 74 \overline{7} 757$

Parameter value

$$
\begin{array}{cc}
0 & 0.0456688 \\
1 & 0.8592813 \\
2 & 0.1 \\
3 & 1.161988
\end{array}
$$

RUNS_COVID/asa_usr_out_21-Kentucky final cost value $=\overline{0} .000224 \overline{6} 308$

Parameter Value

$$
\begin{array}{cc}
0 & 0.03505446 \\
1 & 0.8823249 \\
2 & 0.1 \\
3 & 0.9808715
\end{array}
$$

RUNS COVID/asa usr out 22-Louisiana final cost value $=\overline{0} .000 \overline{8} 01 \overline{5} 797$

Parameter Value

$$
\begin{array}{cc}
0 & 0.1070208 \\
1 & 0.7072564 \\
2 & 2 \\
3 & 0.7402889
\end{array}
$$

RUNS_COVID/asa_usr_out 23-Maine final cost value $=\overline{0} .00 \overline{1} 44 \overline{1506}$

Parameter Value

$$
\begin{array}{ll}
0 & 0.03198315 \\
1 & 0.7940144 \\
2 & 0.1823495 \\
3 & 0.6823531
\end{array}
$$


RUNS COVID/asa usr out 24-Maryland final cost value $=\overline{0} .00 \overline{2} 06 \overline{1062}$

Parameter value

$$
\begin{array}{cc}
0 & 0.0638636 \\
1 & 0.7898237 \\
2 & 0.1 \\
3 & 1.089192
\end{array}
$$

RUNS_COVID/asa_usr_out_25-Massachusetts final cost value $=\overline{0} .00435 \overline{2} 416$

Parameter Value

$$
\begin{array}{cc}
0 & 0.06403747 \\
1 & 0.7364045 \\
2 & 0.1 \\
3 & 1.128749
\end{array}
$$

RUNS_COVID/asa_usr_out_26-Michigan final cost value $=\overline{0} .00 \overline{0} 432 \overline{3} 011$

Parameter Value

$$
\begin{array}{cc}
0 & 0.04372185 \\
1 & 0.7974153 \\
2 & 0.311704 \\
3 & 0.8720471
\end{array}
$$

RUNS_COVID/asa_usr_out_27-Minnesota final cost value $=\overline{0} .0004295167$

$$
\text { Parameter Value }
$$

$$
\begin{array}{cc}
0 & 0.06178572 \\
1 & 0.8006544 \\
2 & 0.1 \\
3 & 1.253828
\end{array}
$$

RUNS_COVID/asa_usr_out_28-Mississippi final cost value $=\overline{0} .00046 \overline{3057}$

Parameter Value

$$
\begin{array}{ll}
0 & 0.1097083 \\
1 & 0.6931913 \\
2 & 0.1054405 \\
3 & 0.9913985
\end{array}
$$

RUNS_COVID/asa_usr_out_29-Missouri final cost value $=\overline{0} .00025 \overline{7} 466$

Parameter Value

$$
\begin{array}{cc}
0 & 0.05215969 \\
1 & 0.8596338 \\
2 & 0.1 \\
3 & 1.055173
\end{array}
$$

RUNS_COVID/asa_usr_out_30-Montana final cost value $=\overline{0} .000442 \overline{8} 111$

Parameter Value

$$
\begin{array}{cc}
0 & 0.03814208 \\
1 & 0.899361 \\
2 & 0.1 \\
3 & 0.9560651
\end{array}
$$

RUNS COVID/asa usr out 31-Nebraska final cost value $=\overline{0} .000326 \overline{7} 622$

Parameter Value

$$
\begin{array}{cc}
0 & 0.04517647 \\
1 & 0.8218373 \\
2 & 0.1 \\
3 & 1.145402
\end{array}
$$

RUNS_COVID/asa_usr_out_32-Nevada final cost value $=\overline{0} .00 \overline{1} 89 \overline{3} 444$

Parameter Value

$$
\begin{array}{cc}
0 & 0.03241173 \\
1 & 0.9219539 \\
2 & 0.1 \\
3 & 1.156847
\end{array}
$$

RUNS_COVID/asa_usr_out_33-New_Hampshire final cost value $=\overline{0} .00 \overline{3} 540 \overline{0} 204$

Parameter Value

$$
\begin{array}{cc}
0 & 0.05990541 \\
1 & 0.713824 \\
2 & 1.999386 \\
3 & 0.5164278
\end{array}
$$

RUNS_COVID/asa_usr_out_34-New_Jersey final cost value $=\overline{0} .00 \overline{3} 76 \overline{4} 219$

Parameter Value

$$
\begin{array}{cc}
0 & 2 \\
1 & 0.3257865 \\
2 & 2 \\
3 & 1.048204
\end{array}
$$

RUNS COVID/asa usr out 35-New Mexico final cost value $=\overline{0} .001152665$

Parameter Value

$$
\begin{array}{cc}
0 & 0.1004785 \\
1 & 0.695894 \\
2 & 0.6817652 \\
3 & 0.7827343
\end{array}
$$

RUNS_COVID/asa_usr out 36-New_York final cost value $=\overline{0} .00 \overline{0} 714 \overline{7} 068$

Parameter Value

$$
\begin{array}{cc}
0 & 0.04110297 \\
1 & 0.7541359 \\
2 & 0.1 \\
3 & 1.054681
\end{array}
$$

RUNS_COVID/asa_usr_out_37-North_Carolina final cost value $=\overline{0} .000 \overline{3} 85 \overline{1} 502$

Parameter value

$$
\begin{array}{cc}
0 & 0.08513204 \\
1 & 0.7664615 \\
2 & 0.1 \\
3 & 1.003618
\end{array}
$$


RUNS_COVID/asa_usr_out_38-North_Dakota final cost value $=\overline{0} .0003929314$

Parameter Value

$$
\begin{array}{cc}
0 & 0.04932907 \\
1 & 0.8614704 \\
2 & 0.1 \\
3 & 0.9475553
\end{array}
$$

RUNS_COVID/asa_usr_out_39-Northern_Mariana_Islands final cost value $=-\overline{0} \overline{1} 10 \overline{5} 92$

Parameter Value

$$
\begin{array}{cc}
0 & 0.03284899 \\
1 & 0.6745235 \\
2 & 0.1 \\
3 & 0.3440228
\end{array}
$$

RUNS COVID/asa usr out 40-Ohio final cost value $=\overline{0} .00 \overline{0} 4090411$

Parameter Value

$\begin{array}{cc}0 & 0.04184926 \\ 1 & 0.8463094 \\ 2 & 0.1 \\ 3 & 1.049081\end{array}$

RUNS_COVID/asa_usr_out_41-Oklahoma final cost value $=\overline{0} .000 \overline{4} 630219$

Parameter Value

$$
\begin{array}{ll}
0 & 0.04501715 \\
1 & 0.8799497 \\
2 & 0.3494903 \\
3 & 0.9048175
\end{array}
$$

RUNS_COVID/asa_usr_out_42-Oregon final cost value $=\overline{0.0009} 20 \overline{8} 029$

Parameter Value

$$
\begin{array}{ll}
0 & 0.05225226 \\
1 & 0.816799 \\
2 & 0.2053155 \\
3 & 0.9100787
\end{array}
$$

RUNS_COVID/asa usr_out 43-Pennsylvania final cost value $=\overline{0} .000 \overline{5} 58 \overline{9} 026$

Parameter Value

$$
\begin{array}{cc}
0 & 0.04241694 \\
1 & 0.8052484 \\
2 & 0.1 \\
3 & 1.015383
\end{array}
$$

RUNS_COVID/asa_usr_out_44-Puerto_Rico final cost value $=\overline{0} .00031 \overline{2} 391$

Parameter Value

$$
\begin{array}{cc}
0 & 0.03449601 \\
1 & 0.9045291 \\
2 & 0.1 \\
3 & 1.088644
\end{array}
$$

RUNS_COVID/asa_usr_out_45-Rhode_Island final cost value $=-\overline{0} 0 . \overline{1111} \overline{4} 74$

Parameter Value

$$
\begin{array}{cc}
0 & 0.04708741 \\
1 & 0.7901072 \\
2 & 0.1 \\
3 & 1.442058
\end{array}
$$

RUNS_COVID/asa_usr_out_46-South_Carolina final cost value $=\overline{0} .00 \overline{0} 972 \overline{2} 008$

Parameter value

$$
\begin{array}{cc}
0 & 0.09290075 \\
1 & 0.7718165 \\
2 & 0.1 \\
3 & 1.095007
\end{array}
$$

RUNS_COVID/asa_usr_out 47-South_Dakota final cost value $=\overline{0} .0003353859$

Parameter Value

$$
\begin{array}{cc}
0 & 0.05975135 \\
1 & 0.7782754 \\
2 & 0.1 \\
3 & 0.9210539
\end{array}
$$

RUNS COVID/asa usr out 48-Tennessee final cost value $=\overline{0} .000517 \overline{8} 384$

Parameter Value

$$
\begin{array}{cc}
0 & 0.09073933 \\
1 & 0.7754924 \\
2 & 2 \\
3 & 0.8461525
\end{array}
$$

RUNS_COVID/asa usr out 49-Texas final cost value $=\overline{0.000 \overline{1}} \overline{\mathbf{1}} \overline{1769}$

Parameter Value

$$
\begin{array}{cc}
0 & 0.05172033 \\
1 & 0.8703259 \\
2 & 0.1 \\
3 & 1.330712
\end{array}
$$

RUNS_COVID/asa_usr_out_50-US final cost value $=\overline{1} .27 \overline{9} 74 \overline{\mathrm{e}}-05$

Parameter Value

$$
\begin{array}{cc}
0 & 0.05285717 \\
1 & 0.8271716 \\
2 & 0.1090954 \\
3 & 1.204249
\end{array}
$$

RUNS_COVID/asa_usr_out_51-Utah final cost value $=\overline{0} 0.00362 \overline{3} 466$

Parameter Value

$$
\begin{array}{cc}
0 & 0.04933961 \\
1 & 0.8573352 \\
2 & 0.1 \\
3 & 1.086935
\end{array}
$$


RUNS_COVID/asa_usr_out_52-Vermont

final cost value $=\overline{0} .000 \overline{8} 160128$

Parameter value

$$
\begin{array}{cc}
0 & 0.006796208 \\
1 & 0.9282152 \\
2 & 0.1 \\
3 & 0.4539584
\end{array}
$$

RUNS_COVID/asa_usr_out_53-Virgin_Islands final cost value $=0.03999473$

Parameter Value

$$
\begin{array}{cc}
0 & 0.06337426 \\
1 & 0.8251611 \\
2 & 0.1 \\
3 & 1.064258
\end{array}
$$

RUNS_COVID/asa_usr_out_54-Virginia final cost value $=\overline{0} .000 \overline{16} \overline{7} 7778$

Parameter Value

$$
\begin{array}{ll}
0 & 0.05090517 \\
1 & 0.8072254 \\
2 & 0.1 \\
3 & 0.9941458
\end{array}
$$

RUNS_COVID/asa_usr_out 55-Washington final cost value $=\overline{0} .000511 \overline{4} 633$

Parameter Value

$$
\begin{array}{cc}
0 & 0.05293824 \\
1 & 0.8114288 \\
2 & 0.1 \\
3 & 1.026359
\end{array}
$$

RUNS_COVID/asa_usr_out_56-West_Virginia final cost value $=\overline{0.0004020269}$

Parameter Value

$$
\begin{array}{cc}
0 & 0.02179989 \\
1 & 0.9542683 \\
2 & 0.1 \\
3 & 0.8805843
\end{array}
$$

RUNS_COVID/asa usr out 57-Wisconsin final cost value $=\overline{0} .000 \overline{5} 233912$

Parameter Value

$$
\begin{array}{cc}
0 & 0.05836374 \\
1 & 0.8373115 \\
2 & 0.1 \\
3 & 1.251952
\end{array}
$$

RUNS_COVID/asa_usr_out_58-Wyoming final cost value $=\overline{0.000} \overline{8048018}$

Parameter Value

$$
\begin{array}{ll}
0 & 0.05755666 \\
1 & 0.7254984 \\
3 & 0.8178418
\end{array}
$$

\section{Conclusion}

Two algorithms are suggested for fitting data and forecasting COVID-19, ASA for importancesampling and fitting parameters to models, and PATHINT/PATHTREE. These algorithms have been applied to several disciplines - neuroscience, financial markets, combat analysis. While optimization and pathintegral algorithms are now quite well-known (at least to many scientists), these previous applications give strong support to application of these tools to COVID-19 data.

\section{ACKNOWLEDGments}

The author thanks the Extreme Science and Engineering Discovery Environment (XSEDE.org), for supercomputer grants since February 2013. The PI has held yearly grants for supercomputer resources from XSEDE, mostly for work on SMNI but for a couple years also to develop qPATHINT and qPATHTREE codes for propagation of quantum wave functions in the presence of random shocks.

\section{References Références Referencias}

Note that some URLs are cited in-text.

1. C. Anastassopoulou, L. Russo, A. Tsakris \& C. Siettos (2020) Data-based analysis, modelling and forecasting of the COVID-19 outbreak. Public Library of Science One. 15(3). [URL https://doi.org/ 10.1371/journal.pone.0230405 ]

2. A.F. Atiya, A.G. Parlos \& L. Ingber (2003) A reinforcement learning method based on adaptive simulated annealing, In: Proceedings International Midwest Symposium on Circuits and Systems (MWCAS), December 2003, IEEE CAS, 121-124. [URL https://www.ingber.com/asa03_reinforce.pdf]

3. I. Bray, A. Gibson \& J. White (2020) Coronavirus disease 2019 mortality: a multivariate ecological analysis in relation to ethnicity, population density, obesity, deprivation and pollution. Public Health. 185, 261-263. [URL https://doi.org/10. 1016/j.puhe.2020.06.056 ]

4. L. Ingber (1993a) Adaptive Simulated Annealing (ASA). Global optimization C-code. Caltech Alumni Association. [URL https://www.ingber.com/\#ASACODE]

5. L. Ingber (1993b) Statistical mechanics of combat and extensions, In: Toward a Science of Command, Control, and Communications, ed. C. Jones. American Institute of Aeronautics and Astronautics, 117-149. [ISBN 1-56347-068-3. URL https://www. ingber.com/combat93_c3sci.pdf]

6. L. Ingber (1996) Canonical momenta indicators of financial markets and neocortical EEG, In: Progress in Neural Information Processing, ed. S.-I. Amari,L. Xu, I. King \& K.-S. Leung. Springer, 777-784. [Invited paper to the 1996 International 
Conference on Neural Information Processing (ICONIP'96), Hong Kong, 24-27 September 1996. ISBN 981-3083-05-0. URL https://www.ingber.com/markets96_momenta. pdf]

7. L. Ingber (2010) Trading in Risk Dimensions, In: The Handbook of Trading: Strategies for Navigating and Profiting from Currency, Bond, and Stock Markets, ed. G.N. Gregoriou. McGraw-Hill, 287300.

8. L. Ingber (2012) Adaptive Simulated Annealing, In: Stochastic global optimization and its applications with fuzzy adaptive simulated annealing, ed. H.A. Oliveira, Jr., A. Petraglia, L. Ingber, M.A.S. Machado \& M.R. Petraglia. Springer, 33-61. [Invited Paper. URL https://www.Ingber.com/asa 11_options.pdf ]

9. L. Ingber (2015) Biological Impact on Military Intelligence: Application or Metaphor?. International Journal of Intelligent Defence Support Systems. 5 (3), 173-185. [URL https://www.ingber.com/ combat15 milint.pdf ]

10. L. Ingbēer (2018) Quantum calcium-ion interactions with EEG. Sci. 1(7), 1-21. [URL https://www.ingber.com/smni18_quantumCaEEG. pdf and https://doi.org/10.3390/sci1010020]

11. L. Ingber (2020a) Developing bid-ask probabilities for high-frequency trading. Virtual Economics. 3(2), 7-24. [URL https://www.ingber.com/markets19_bid ask_prob.pdf and https://doi.org/10.34021/ve.202 0.03.02(1)]

12. L. Ingber (2020b) Forecasting with importancesampling and path-integrals: Applications to COVID-19. Report 2020: FISPI. Physical Studies Institute. [URL https://www.ingber.com/asa20_fore cast.pdf and https://doi.org/10.20944/preprints2020 09.0385.v3 ]

13. L. Ingber (2021) Quantum calcium-ion affective influences measured by EEG. Int. J. Physics Space Science., (to be published). [URL https:// www.ingber.com/quantum20_affective.pdf and https://doi.org/10.20944/preprints202009.0591.v1 ]

14. L. Ingber \& R.P. Mondescu (2001) Optimization of trading physics models of markets. IEEE Transactions Neural Networks. 12(4), 776-790. [Invited paper for special issue on Neural Networks in Financial Engineering. URL https://www.ingber. com/markets01_optim_trading.pdf]

15. L. Ingber \& R.P. Mondescu (2003) Automated internet trading based on optimized physics models of markets, In: Intelligent Internet-Based Information Processing Systems, ed. R.J. Howlett, N.S. Ichalkaranje, L.C. Jain \& G. Tonfoni. World Scientific, 305-356. [Invited paper. URL https:// www.ingber.com/markets03_automated.pdf]

16. A.Y. Li, T.C. Hannah, J. Durbin, N. Dreher, F.M. McAuley, N. F. Marayati, Z. Spiera, M. Ali, A. Gometz, J.T. Kostman \& T.F. Choudhri (2020)
Multivariate analysis of black race and environmental temperature on COVID-19 in the US. The American Journal of the Medical Sciences. 360(4), 348-356. [URL https://doi.org/10.1016/j. amjms.2020.06.015 ]

17. C. Okoli \& S.D. Pawlowski (2004) The Delphi method as a research tool: an example, design considerations and applications. Information and Management. 42(1), 15-29. [URL https://doi. org/10.1016/j.im.2003.11.002] 